\title{
BMJ Open How European primary care practitioners think the timeliness of cancer diagnosis can be improved: a thematic analysis
}

\author{
Michael Harris (10 , ${ }^{1,2}$ Hans Thulesius, ${ }^{3,4}$ Ana Luísa Neves, ${ }^{5,6}$ Sophie Harker, ${ }^{1}$ \\ Tuomas Koskela, ${ }^{7}$ Davorina Petek, ${ }^{8}$ Robert Hoffman (1) , ${ }^{9}$ Mette Brekke, ${ }^{10}$ \\ Krzysztof Buczkowski, ${ }^{11}$ Nicola Buono (1) , ${ }^{12}$ Emiliana Costiug, ${ }^{13}$ \\ Geert-Jan Dinant, ${ }^{14}$ Gergana Foreva, ${ }^{15}$ Eva Jakob, ${ }^{16}$ Mercè Marzo-Castillejo, ${ }^{17}$ \\ Peter Murchie (1) , ${ }^{18}$ Jolanta Sawicka-Powierza, ${ }^{19}$ Antonius Schneider, ${ }^{20}$ \\ Emmanouil Smyrnakis, ${ }^{21}$ Sven Streit, ${ }^{2}$ Gordon Taylor, ${ }^{22}$ Peter Vedsted, ${ }^{23}$ \\ Birgitta Weltermann, ${ }^{24}$ Magdalena Esteva ${ }^{25}$
}

To cite: Harris M, Thulesius $\mathrm{H}$, Neves AL, et al. How European primary care practitioners think the timeliness of cancer diagnosis can be improved: a thematic analysis. BMJ Open 2019;9:e030169. doi:10.1136/ bmjopen-2019-030169

- Prepublication history and additional material for this paper are available online. To view these files, please visit the journal online (http://dx.doi org/10.1136/bmjopen-2019030169).

Received 04 March 2019 Revised 17 July 2019 Accepted 10 September 2019

D Check for updates

(c) Author(s) (or their employer(s)) 2019. Re-use permitted under CC BY-NC. No commercial re-use. See rights and permissions. Published by BMJ.

For numbered affiliations see end of article.

\section{Correspondence to}

Professor Michael Harris; michaelharris681@btinternet. com

\section{ABSTRACT}

Background National European cancer survival rates vary widely. Prolonged diagnostic intervals are thought to be a key factor in explaining these variations. Primary care practitioners (PCPs) frequently play a crucial role during initial cancer diagnosis; their knowledge could be used to improve the planning of more effective approaches to earlier cancer diagnosis.

Objectives This study sought the views of PCPs from across Europe on how they thought the timeliness of cancer diagnosis could be improved.

Design In an online survey, a final open-ended question asked PCPs how they thought the speed of diagnosis of cancer in primary care could be improved. Thematic analysis was used to analyse the data.

Setting A primary care study, with participating centres in 20 European countries.

Participants A total of 1352 PCPs answered the final survey question, with a median of 48 per country.

Results The main themes identified were: patient-related factors, including health education; care provider-related factors, including continuing medical education; improving communication and interprofessional partnership, particularly between primary and secondary care; factors relating to health system organisation and policies, including improving access to healthcare; easier primary care access to diagnostic tests; and use of information technology. Re-allocation of funding to support timely diagnosis was seen as an issue affecting all of these. Conclusions To achieve more timely cancer diagnosis, health systems need to facilitate earlier patient presentation through education and better access to care, have well-educated clinicians with good access to investigations and better information technology, and adequate primary care cancer diagnostic pathway funding.

\section{INTRODUCTION}

Cancer survival rates vary widely in Europe, ${ }^{1}$ leading to considerable additional mortality

\section{Strengths and limitations of this study}

We recruited primary care practitioners (PCPs) from 20 European countries, 4 countries from each of the Northern, Southern, Eastern, Western and Central European geographical areas, to provide variation in geography, health systems and levels of healthcare spending.

- The recruitment method allowed participation of physicians in countries in which PCPs are under-represented in research.

- The survey had only one question that related directly to PCPs' views on the timeliness of cancer diagnosis.

- Neither patients, secondary care nor other primary care health professionals were included in the survey, and these groups may have had other insights to offer.

in some countries. Data from the European Cancer Registry-based Study on Survival and Care of Cancer Patients (EUROCARE-5) show that the national 1-year relative survival rates for all cancer sites vary from $58.2 \%$ to $81.1 \%{ }^{2}$ Poorer 1-year relative survival is thought to be indicative of diagnostic delay ${ }^{34}$ and more advanced disease at diagnosis, ${ }^{56}$ although it can be affected by differences in registration, as well as overdiagnosis and lead-time biases. ${ }^{78}$ There may also be geographical differences in the incidence of cancers that have a poorer prognosis, ${ }^{9}$ as well as national variations in access to effective cancer treatment, ${ }^{10}$ and in levels of poverty, which in itself has been linked with more advanced disease stage at diagnosis. ${ }^{11-13}$ More advanced cancers are more difficult to treat successfully ${ }^{14}$ and, for 
many cancers, the stage of disease at diagnosis is related to survival. ${ }^{1516}$ There is considerable evidence that longer time to diagnosis and treatment has an adverse impact on cancer mortality. ${ }^{17-23}$ Timely care also adds to patients' quality of life and emotional well-being. ${ }^{24}$ In addition, patients' descriptions of the quality of their care are closely related to the speed of their diagnosis and treatment; this is because they are concerned that longer waits might allow time for their cancers to grow. ${ }^{25}$

Timely diagnosis of cancer has therefore been an important aim of healthcare providers across Europe. ${ }^{25}$ However, the challenge in deciding where and how to achieve this is substantial. ${ }^{26}$ In countries where a comparatively low 1-year cancer survival suggests that late diagnosis may be a major factor, it is uncertain whether this is due to patients presenting later to healthcare, whether they are not being referred quickly enough by those in primary care, or whether they are not being investigated and managed efficiently in secondary care. ${ }^{14}$ This may be a particular problem where patients with cancer present without red-flag symptoms, as how their primary care practitioners (PCPs) act depends on how their health systems are organised. ${ }^{27}$

Doctors and their patients recognise that general practitioners (GPs) and other PCPs have a key role in cancer detection. ${ }^{28-30}$ It has been suggested that PCPs' knowledge of their patient populations can be used to improve health service design ${ }^{31}$ and the planning for more effective approaches to earlier cancer recognition and referral. ${ }^{32}$ There has been a call for research in this field $^{15}$; although PCPs' experience of their own health systems could potentially help to improve the planning of more effective approaches to earlier cancer recognition and referral, their perceptions have not been previously evaluated.

The aim of this study was to elicit the views of GPs and other PCPs from across Europe on how they thought the timeliness of cancer diagnosis could be improved.

\section{METHODS AND DESIGN \\ Design}

Between November 2015 and December 2016, we performed an online survey of PCPs in 20 European countries. The methodology of the study is described in our published protocol paper. ${ }^{33}$

\section{Development of the questionnaire}

The Örenäs Research Group is a European group of primary care researchers that studies the primary care factors that relate to cancer survival. After a literature review, Örenäs Research Group investigators developed a questionnaire designed to elicit PCPs' referral decisions on patients who could have cancer, and their degree of agreement with items relating to health system aspects that could affect their decision to refer patients with potential cancer symptoms for further investigation. The final, open-ended question asked: "How do you think the speed of diagnosis of cancer in primary care could be improved?" The answers to this question are used in this analysis.

The questionnaire was piloted twice by PCPs in 16 Örenäs Research Group centres. No changes were made to the final, open-ended question after either of the piloting stages. Örenäs Research Group leads arranged for translations of the questionnaire into their local languages where these were not English, a total of 19 translations from the original English. Translation, validation by back-translation to assess semantic and conceptual equivalence, and cultural adaptation ${ }^{34}$ of the survey were done in a standardised way ${ }^{35}$ and are described elsewhere. ${ }^{36}$ The questionnaires were put online using SurveyMonkey (SurveyMonkey, California, USA).

\section{Participants and recruitment}

The study was conducted in 25 Örenäs Research Group centres in 20 countries across Europe: Bulgaria, Croatia, Denmark, England, Finland, France, Germany, Greece, Israel, Italy, The Netherlands, Norway, Poland, Portugal, Romania, Scotland, Slovenia, Spain, Sweden and Switzerland. Local study leads were asked to either gain ethical approval or obtain a statement that formal ethical approval was not needed in their jurisdiction (see online supplementary file).

Criterion sampling was used, ${ }^{37}$ with subjects being eligible for the survey if they were doctors working mainly in primary care. These doctors, referred to collectively here as 'PCPs', consisted of GPs as well as other doctors who had specialist training but worked in the community and could be accessed directly by patients without referral.

Each Örenäs Research Group local lead was asked to email a survey invitation to the PCPs in their local health district, and to recruit at least 50 participants, with no maximum limit. This allowed recruitment of a varied sample with regard to gender, years since graduation, site of practice (urban, rural, remote) and size of practice. Consent was implied by agreeing to take part in the survey.

\section{Data analysis}

Local Örenäs Research Group leads translated the responses from their own languages into English and sent them to MH, who asked them for more information where the translation or context was unclear.

We used inductive thematic analysis, ${ }^{38}$ an approach in which codes and themes are suggested by the data rather than by a theoretical framework. The phases of analysis included coding, followed by the identification and clustering of themes and subthemes, and the production of a descriptive thematic summary. Two researchers (MH and $\mathrm{SH})$ independently coded the data from three countries and compared their analyses for inconsistencies and agreement. MH then coded the data from the other countries. Team members from seven participating countries (ME, MH, RH, TK, ALN, DP, HT, all PCPs) then independently 
Table 1 National distribution of primary care practitioners (PCPs) who responded to the question "How do you think the speed of diagnosis of cancer in primary care could be improved?"

\begin{tabular}{lcc}
\hline Country & $\begin{array}{l}\text { Number of PCPs } \\
\text { who completed } \\
\text { the survey }\end{array}$ & $\begin{array}{l}\text { Number who answered } \\
\text { the open-ended } \\
\text { question (\% of all survey } \\
\text { completers) }\end{array}$ \\
\hline Bulgaria & 52 & $45(86.5)$ \\
\hline Croatia & 56 & $42(75.0)$ \\
\hline Denmark & 92 & $71(77.2)$ \\
\hline England & 62 & $25(40.3)$ \\
\hline Finland & 61 & $39(63.9)$ \\
\hline France & 52 & $35(67.3)$ \\
\hline Germany & 91 & $31(34.1)$ \\
Greece & 59 & $50(84.7)$ \\
\hline Israel & 58 & $42(72.4)$ \\
\hline Italy & 60 & $52(86.7)$ \\
\hline The & 108 & $84(77.8)$ \\
Netherlands & & $46(56.8)$ \\
\hline Norway & 81 & $103(76.3)$ \\
\hline Poland & 135 & $46(78.0)$ \\
\hline Portugal & 59 & $132(90.4)$ \\
\hline Romania & 146 & $55(88.7)$ \\
\hline Scotland & 62 & $52(57.1)$ \\
\hline Slovenia & 91 & $332(87.4)$ \\
\hline Spain & 380 & $55(80.9)$ \\
\hline Sweden & 68 & $1525.0)$ \\
\hline Switzerland & 60 & $73.8)$ \\
\hline Total & 1833 & \\
\hline & & \\
\hline
\end{tabular}

considered themes and subthemes, discussed these and came to a consensus over the course of two meetings. The themes and subthemes were grouped to construct an interpretative narrative across the dataset and depicted diagrammatically. Apart from SH, a Masters psychology student, all authors involved in the coding and thematic analysis were experienced GPs who were also active in primary care research.

\section{Patient and public involvement}

There was no patient or public involvement in this study.

\section{RESULTS}

A total of 1833 PCPs completed the full questionnaire, with a median response rate per country of $24.8 \%$ (range $7.1 \%-65.6 \%$ ). All participating centres received at least 50 responses, with a median of 61 PCPs per country. In all, 1352 PCPs ( $73.8 \%$ of completers) gave an answer to the final, open-ended survey question 'How could the speed of diagnosis of cancer in primary care could be improved?', with a median of 48 per country (table 1 ).
Table 2 Demographic distribution of primary care practitioners who responded to the question "How do you think the speed of diagnosis of cancer in primary care could be improved?"

\begin{tabular}{lc}
\hline Demographic & Number (\%) \\
\hline $\begin{array}{l}\text { Gender } \\
\text { Female }\end{array}$ & $833(61.6)$ \\
\hline Male & $513(38.0)$ \\
\hline Not stated & $5(0.4)$ \\
Years since graduation & \\
\hline$<10$ & $192(14.2)$ \\
\hline $10-19$ & $356(26.4)$ \\
\hline $20-29$ & $416(30.8)$ \\
\hline $30-39$ & $336(24.9)$ \\
\hline 40 or over & $47(3.5)$ \\
\hline Not stated & $4(0.3)$ \\
\hline Site of practice & \\
\hline Urban & $816(60.4)$ \\
\hline Rural & $314(23.2)$ \\
\hline Island & $25(1.9)$ \\
\hline Mixed & $194(14.4)$ \\
\hline Not stated & $2(0.1)$ \\
\hline Number of doctors in practice & \\
\hline $1-2$ & $337(24.9)$ \\
\hline $3-5$ & $344(25.5)$ \\
\hline $6-9$ & $290(21.5)$ \\
\hline 10 or more & $374(27.7)$ \\
\hline Not stated & $6(0.4)$ \\
\hline
\end{tabular}

To reduce the risk of bias from countries with larger numbers of respondents, we coded a maximum of 100 respondents' comments, randomly sampled, per country. The demographic distributions of the PCPs answering this final survey question are shown in table 2. Towards the end of the analysis, no new themes emerged.

We identified four main themes organising the content of the responses to the final survey question: patient-related factors, care provider-related features, improving communication and interprofessional partnership and aspects of health system organisation and health policies. Two structural facets transcended the four themes: accessing diagnostic tests and using information technology. The issue of allocating funding to support timely diagnosis fed into all the other themes. The themes are shown diagrammatically in figure 1 . Themes and subthemes are described below, with participant quotations identified by country and a participant number.

\section{Patient-related factors}

This theme included ideas on education and training to improve patient knowledge. Many PCPs advocated the 


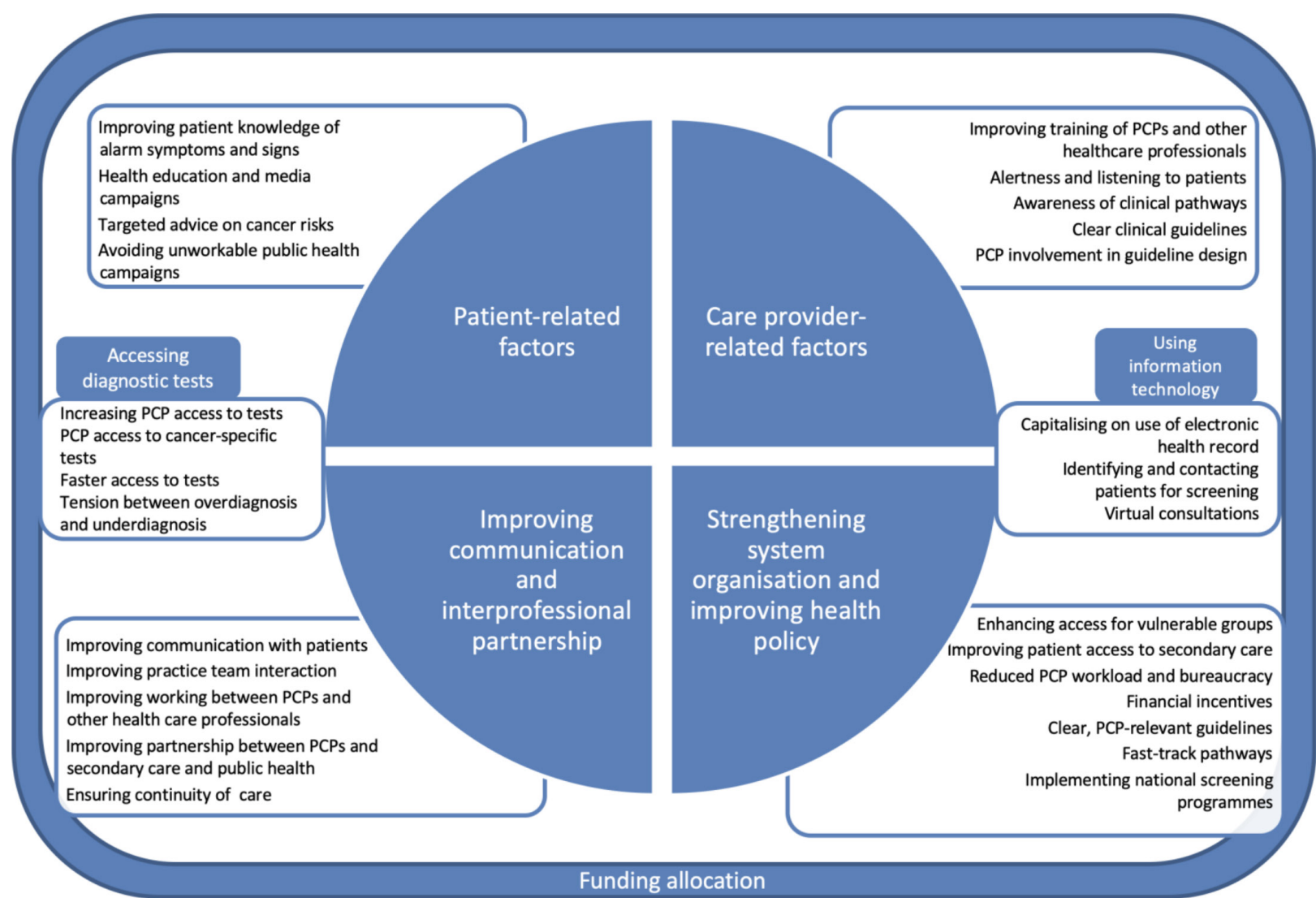

Figure 1 Diagrammatic representation of themes emerging from responses to the question "How do you think the speed of diagnosis of cancer in primary care could be improved?" PCP, primary care practitioner.

use of health education and media campaigns to improve patient awareness of alarm symptoms and signs:

By giving patients better information about when to go to a doctor-reduce patient delay. (Denmark, PCP69)

Improve health literacy of patients regarding the detection of early symptoms that might indicate oncological disease. (Portugal, PCP37)

Targeting advice on cancer risks was also considered important:

Informing patients about cancer risks related to their age groups. (Italy, PCP28)

However, public health messages could be seen as unworkable:

Stop public campaigns telling everyone with a particular symptom they need for example, a chest X-ray after 3 weeks of cough-inappropriately blocking the service. (Scotland, PCP16)

\section{Care provider-related factors}

PCPs had suggestions on how to improve their own knowledge and that of other caregivers, by both increasing the amount of the training and improving the teaching content. Improving the training of PCPs and other healthcare professionals was considered by many respondents to be important:

By educating healthcare professionals. When to suspect and when to do further tests? (Finland, PCP33)

CME [continuing medical education] on early signs and up-to-date investigation processes. (Sweden, PCP52)

Alertness in their role was also considered key:

Often the presence of alarm signs is too late ... Be alert and listen to the patients. (Spain, PCP46)

Primary care doctors also need to be aware of the relevant clinical pathways:

Training of GPs ... taking into account the entire pathway from symptom to diagnosis. (Poland, PCP17)

PCPs thought it important that cancer screening and referral guidelines should be clear, and that they themselves should be involved in designing them.

Clear guidelines regarding cancer alarm signs and referral indications. (Finland, PCP37)

GPs who design guidelines together with specialists. (Sweden, PCP21) 
Improving communication and interprofessional partnership

Patient-PCP communication improvements and enhancements to interprofessional partnerships were frequently suggested by the PCP respondents. Improving communication with patients was considered important, although this implied having longer consultations:

GPs should pay more attention when they speak to patients. (Italy, PCP26)

Longer consultations to allow more thorough assessment without pressure of time. (Scotland, PCP5)

Improving the interaction within the practice team could also be helpful:

Discussion in the team about recent referrals so the team benchmarks itself. (England, PCP22)

A lot can be learnt from colleagues' experiences of missed diagnoses. (The Netherlands, PCP40)

Improving partnership between PCPs and other primary healthcare professionals, for example, nurses and healthcare assistants, with adequate training, was also considered important:

Involve the nurses in gathering the patient's medical history. (Poland, PCP20)

Good training of healthcare assistant to identify risk symptoms. (Norway, PCP1)

Improving the relationship between primary and secondary care doctors, with easier and reliable communication between the two, was highlighted:

Easy communication with specialists (and, in my case, preferably email contact). (The Netherlands, PCP85)

It is essential to establish a communication channel between primary healthcare and specialist healthcare so that critical patients would not get lost in the system. (Croatia, PCP36)

Joint working with public health departments was also considered valuable:

With the cooperation between the National Institute of Public Health and family medicine specialists, who know their patients best. (Croatia, PCP38)

Many doctors commented on the importance of continuity of care:

Knowing your patient well. Consequently, not having three different GPs for one patient. (The Netherlands, PCP69)

\section{Strengthening system organisation and improving health policy}

Strengthening how health systems are organised and implementing health policies that allow timely cancer diagnosis were key recommendations from many PCPs. Enhancing patient access to primary care, particularly for vulnerable or more remote groups, was considered important by some:

Shorter queues to GP appointment in order to start assessments without delay. (Finland, PCP33)

In some places (rural, inland) inaccessibility to MCDTs [screening examinations] can be an obstacle. Portugal, PCP43)

Practical steps to improve access to secondary care were also seen as key, particularly those relating to costs to the patient:

Access to specialists must be cheaper. (Romania, PCP99)

Patient-friendly hospital services-so that the appointments are not too stressful or expensive (travel and parking). (England, PCP19)

Many PCPs commented on the need for better working conditions, a larger workforce, a reduced workload and less bureaucracy:

A sufficient number of healthcare professionals so that there is not too much workload for a few. (Croatia, PCP5)

Reduction of the workload at the family medicine doctor office. (Croatia, PCP31)

By decreasing bureaucracy! So we will have more time for a real consultation! (Romania, PCP49)

The adoption of financial incentives schemes was also suggested:

Allocating additional financial resources and stimulating the GP in this direction. (Romania, PCP78)

Financial incentives for preventative care of most common malignant conditions. (Bulgaria, PCP4)

PCPs felt that guidelines and protocols needed to be clear and relevant to them:

Clear guidelines regarding cancer alarm signs and referral indications. (Finland, PCP37)

By developing a more specific GP protocol for the various types of cancer. (The Netherlands, PCP51)

Fast-track systems for patients with cancer were requested by many respondents:

Create a fast-track unit for patients with high cancer suspicion. (Spain, PCP51)

In countries where fast-track systems already existed, these were seen as a success:

Cancer diagnostics have priority in the Norwegian healthcare system since fast-track referral was introduced last year. Functions well. (Norway, PCP5)

I actually think that the introduction of the " 2 week wait" urgent referral system has greatly improved the 
speed of diagnosis for those with obvious red flag symptoms. (England, PCP11)

Some PCPs felt that there should be more uptake of national screening programmes:

Encourage patients to use existing prevention programmes. (Germany, PCP7)

All physicians should adhere to screening guidelines. (Switzerland, PCP8)

\section{Accessing diagnostic tests}

This theme suggesting improvements to diagnostic structures dealt mainly with easier and quicker access to testing. Easier PCP access to diagnostic testing was cited by many respondents:

Increasing the range of diagnostics available; also making them easier to refer for. (The Netherlands, PCP79)

Facilitating family doctors to request tests and investigations that he/she considers necessary for the diagnosis. (Spain, PCP39)

Many felt that they should have direct access to cancer-specific testing:

GPs should be able to recommend any investigation without reference to other specialist doctors. (Romania, PCP44)

Give GPs the ability to refer patients to more diagnostic tests, for example, CT, tumour markers. (Poland, PCP26)

The speed of access to diagnostic tests was also seen as key:

Faster access/fast track to imaging diagnostics could help us exclude a number of cases and so could relieve specialist services. (Norway, PCP18)

Reduction of the delay in getting special tests. (France, PCP 2)

However, some PCPs were worried that the increase in investigation of patients needed to diagnose cancers earlier could also lead to overdiagnosis and overtreatment:

Cancer diagnostics are a difficult balancing act between under- and overdiagnosis. Faster cancer diagnostics will also give more overdiagnosis. (Norway, PCP 31)

Depends on the type of cancer, but there is a trade-off between diagnosing a few cancers earlier by referring lots more patients to specialist clinics and the increased stress, anxiety, expense and risk of physical harm from unnecessary ionising radiation and treatments. (England, PCP 7)

\section{Using information technology}

This theme was about using electronic information and communication to improve diagnostic capability and enhance partnership working. Some PCPs thought that more decision support aids should be included in electronic health records:

Automatic decision support/diagnostic support in the GP electronic information system based on symptoms. (The Netherlands, PCP10)

The 29 Norwegian guidelines regarding "fast track referral for cancer" could be included into our electronic patient files as a real decision aid with extraction of clinical information for the referral. (Norway, PCP3)

Information technology could also be used to help identify those who would benefit from screening, and provide reminders relating to individual patients where needed:

Select specific age groups from healthcare databases and identify the individuals that haven't had the screening. (Portugal, PCP11)

Using an automatic reminder system for cancer screening for each patient. (Greece, PCP15)

Some PCPs were keen on the option to have "virtual consultations" with specialists:

\section{Allocating funding to support timely diagnosis}

Adequate resources for primary care and targeted funding for investigations were frequently recommended by participants. This theme wove through the whole cancer diagnostic process. An increase in primary care financing was considered to be necessary if PCPs were to improve access to care:

By increasing resources in primary care in order to improve the access to care. (Finland, PCP34)

Adequate funds were also seen to be needed for screening and diagnostic tests:

Increase funding of screening tests. (Poland, PCP20) A higher budget for diagnostic tests, covering ... some higher costs related to the investigation and early diagnosis of cancer. (Romania, PCP59)

In some countries, there was a need for an increase in the budget for diagnostic tests and referrals:

Increase funding for cancer diagnostic tests (tumour markers, colonoscopy, gastroscopy, radiographs)currently, the funding is insufficient. (Poland, PCP29)

Unlimited lab tests and unlimited referrals to specialist services if malignant conditions are suspected. (Bulgaria, PCP11)

Funding limitations could be an issue for patients:

Many patients refuse the investigations because of the costs (tumour markers, MRI, extra payment to the specialist doctor, ineffective collaboration between specialities). (Romania, PCP12) 
This could also affect the finances of the PCPs themselves:

Ensure adequate financial support-at present there are penalties if the number of consultations exceeds the limit, even if the consultations are clinically indicated. (Bulgaria, PCP25)

\section{DISCUSSION}

\section{Principal findings}

In this primary care study, participants from 20 countries were able to use their experience situated in their own healthcare systems to provide a rich variety of suggestions for the improvements needed to allow more timely diagnosis of cancer. These covered the whole diagnostic pathway, from recommendations on how to help patients to present earlier with their symptoms, through continuing medical education that would help PCPs to recognise and act on symptoms that could be due to cancer, to improving communication and interprofessional partnership between and within primary and secondary care. Improving health system organisation and health policies is essential for achieving these goals. Facilitating patients' access to healthcare was also considered important, as was enabling fast and direct PCP access to diagnostic tests. Respondents saw funding allocation as an underlying component of most of these issues. They also identified a risk that reducing delays in cancer diagnosis in some patients could lead to overdiagnosis in others.

\section{Strengths and weaknesses of the study}

This is the first trans-European study to explore PCPs' perceptions of how timeliness of cancer diagnosis can be improved, offering a unique insight into the operational and administrative challenges. This could be invaluable for the evaluation and revision of current healthcare policies and practices. Cancer diagnosis is a complex process and the diagnostic behaviour of PCPs working under the influences of their native healthcare systems is a key determinant. A key strength of our work is that we have gathered the views of PCPs from 20 countries in this process; our analysis examines systemic influences on PCP cancer diagnostic activity across Europe.

Variation in geography, health systems and levels of healthcare spending was provided by having four participating countries from each of the Central, Eastern, Northern, Southern and Western European geographical areas. The sample size was large and diverse, with participants varying in terms of years of clinical practice, gender and site and size of practice. The study successfully recruited participants in countries in which PCPs are under-represented in research.

The survey had only one, short question that related directly to our research question. However, it may be that this format prompted participants to focus on writing down only what, for them, was the most important point. Neither patients, secondary care nor other PCPs were included in the survey, and these groups may have had other insights to offer. There is a risk that the translators and investigators misunderstood some of the nuances of the 20 different languages, cultures and healthcare organisations involved in this study. While themes were identified from responses from PCPs in 20 European countries, not all of the themes identified will be equally relevant to each of the participating countries.

The data collection was completed in December 2016, and participating countries' health systems will have continued to develop since then. However, each of the themes results from PCPs' comments on healthcare is situated in a society and culture which changes very slowly, as is also seen for implementation of new knowledge. The findings are therefore likely to continue to be relevant.

\section{Comparison with other studies}

Our findings reflect those of a survey of GPs in Ireland, which identified that barriers to early cancer diagnosis included lack of direct GP access to diagnostic tests, difficulties with referral to secondary care, poor clarity relating to cancer screening, unequal patient access to secondary services and a need for further training and guidelines. ${ }^{39}$ GPs' views on the importance of closer links between primary and secondary care were identified in that and other studies. ${ }^{29} 30$ Our PCPs' call for improved patient education and better access to diagnostic testing specialist care were also prioritised by GPs in a UK study. ${ }^{40}$ Our findings map across to the findings from qualitative research on patient-perceived barriers to early presentation and diagnosis of cancer, which have identified the need for better access to services for patients ${ }^{41} 42$ and better patient awareness of cancer symptoms ${ }^{42}$ as key issues. Our PCPs' call for an improved partnership with other primary healthcare professionals is one that has been successfully implemented before, although not specifically in relation to cancer diagnosis. ${ }^{43}$ Their recommendation for better cancer awareness among primary care staff is confirmed by evidence from other research that there is a need for this. ${ }^{44}$

GPs in other studies have identified issues that were not raised by PCPs in our study, including the perception that late presentation of cancer symptoms often relates to patients' socioeconomic and sociocultural circumstances, ${ }^{15} 30$ a concern that fragmentation of health services adversely affects timeliness of cancer diagnosis, ${ }^{154}$ and a need for mentoring of doctors unfamiliar with their health systems. ${ }^{30}$

The need for healthcare systems to support PCPs' quick and easy access to investigations has been proposed before. ${ }^{46}$ While investigation in primary care has been linked with later referral for specialist assessment, reducing the waiting time for tests would be expected to shorten the primary care intervals associated with investigation use. ${ }^{47}$ Despite this, another study found that some patients received a delayed cancer diagnosis, even when they had presented with typical cancer symptoms to a GP who had access to relevant diagnostic tests. ${ }^{48}$ 


\section{Possible implications for clinicians and policymakers}

PCP respondents had specific recommendations on the changes that are needed to facilitate more timely diagnosis of cancer. Some recommendations, for instance health education campaigns and development of relevant guidelines, may need central direction, although with the input of PCPs. Others, for example improving the way PCPs communicate with secondary care specialists, and PCPs' ability to access to cancer-specific tests, may need local agreement. Aspects such as PCPs' own communication skills, their own accessibility to patients and their continuing medical education, are more likely to be under PCPs' own control. However, a consideration of how funding is best reallocated is crucial if PCPs and their health systems are able to make these changes.

\section{Unanswered questions and future research}

Further analysis is needed to help identify which recommendations are most relevant to different existing models of healthcare, for example as to whether some are particularly relevant to systems in which the PCP has more of a "gatekeeper" role, or to those in which PCP practises are large or smaller than average. A longitudinal study would give evidence on the trends on PCP opinions and how they are impacted by changes in health policies and public health initiatives. Research using interviews would give in-depth ideas on how changes resulting from the study findings could be implemented. A mixed-methods approach, comparing PCPs' views from different countries, would allow recommendations that are relevant to individual countries. In the study we have focused on the view of PCPs, as they have a key role in cancer diagnosis throughout Europe; however, the views of other stakeholders, such as patients and policy makers, are also important and should be included in future qualitative research.

There is a need to consider how to get the best balance between achieving early cancer diagnosis and minimising overdiagnosis of cancer. Our study gives the basic elements for the international primary care cancer research agenda, which would provide a knowledge base for developing and improving cancer diagnosis and prognosis internationally.

\section{CONCLUSIONS}

This research has identified key features that PCPs believe would be necessary to improve the timeliness of cancer diagnosis in their patients, and a need for re-allocation of health system funding to allow these to happen. Health systems need to facilitate earlier patient presentation through health education and better access to care, have well-educated clinicians with good access to investigations and better information technology, and adequate primary care cancer diagnostic pathway funding.

Many PCPs play a crucial role at the centre of their patients' care, and the breadth of their experience is reflected in their wide range of suggestions. This understanding should help to inform health service policy and research towards better cancer outcomes.

\section{Author affiliations}

${ }^{1}$ Department for Health, University of Bath, Bath, UK

${ }^{2}$ Berner Institut für Hausarztmedizin (BIHAM), University of Bern, Bern, Switzerland

${ }^{3}$ Department of Clinical Sciences, Lund University, Lund, Sweden

${ }^{4}$ Department of Research and Development, Region Kronoberg, Sweden

Institute of Global Health Innovation, Imperial College London, London, UK

${ }^{6}$ CINTESIS (Centre for Health Technology and Services Research) and MEDCIDS

(Department of Community Medicine, Information and Health Decision Sciences),

Faculty of Medicine, University of Porto, Porto, Portugal

${ }^{7}$ Faculty of Medicine and Health Technology, Tampere University, Tampere, Finland

${ }^{8}$ Department of Family Medicine, University of Ljubljana, Ljubljana, Slovenia

${ }^{9}$ Department of Family Medicine, Tel Aviv University, Tel Aviv, Israel

${ }^{10}$ Department of General Practice and General Practice Research Unit, University of 0slo, Oslo, Norway

${ }^{11}$ Department of Family Medicine, Nicolaus Copernicus University, Toruń, Poland

${ }^{12}$ Department of General Practice, National Society of Medical Education in General

Practice (SNaMID), Caserta, Italy

${ }^{13}$ Family Medicine Department, luliu Hatieganu University of Medicine and

Pharmacy, Cluj-Napoca, Romania

${ }^{14}$ Department of General Practice, Maastricht University, Maastricht, The Netherlands

${ }^{15}$ Medical Center BROD, Plovdiv, Bulgaria

${ }^{16}$ Primary Health Centre, Centro de Saúde Sarria, Sarria, Lugo, Spain

${ }^{17}$ Unitat de Suport a la Recerca, IDIAP Jordi Gol, Institut Català de la Salut,

Barcelona, Spain

${ }^{18}$ Division of Applied Health Sciences - Academic Primary Care, University of Aberdeen, Aberdeen, UK

${ }^{19}$ Department of Family Medicine, Medical University of Bialystok, Bialystok, Poland

${ }^{20}$ TUM School of Medicine, Institute of General Practice and Health Services

Research, Technical University of Munich, München, Germany

${ }^{21}$ Laboratory of Primary Health Care, General Practice and Health Services

Research, Aristotle University of Thessaloniki, Thessaloniki, Greece

${ }^{22}$ College of Medicine and Health, University of Exeter, Exeter, UK

${ }^{23}$ Research Unit for General Practice, Aarhus University, Aarhus, Denmark

${ }^{24}$ Institut für Hausarztmedizin, University of Bonn, Bonn, Germany

${ }^{25}$ Research Unit, Majorca Primary Health Care Department, Balearic Islands Health Research Institute (IdISBa), Preventive Activities and Health Promotion Network, Carlos III Institute of Health (RedIAPP-RETICS), Palma de Mallorca, Spain

Correction notice This article has been corrected since it was published. Author name has been updated.

Acknowledgements The authors would like to thank all the PCPs who piloted the questionnaire and those who completed the survey. The authors would also like to thank the following non-author contributors: Isabelle Aubin-Auger (Université Paris Diderot, Paris, France), Joseph Azuri (Tel Aviv University, Tel Aviv, Israel), Svjetlana Gašparović Babić (The Teaching Institute of Public Health of Primorsko-goranska County, Rijeka, Croatia) and Marija Petek Šter (University of Ljubljana, Ljubljana, Slovenia).

Contributors KB, MB, NB, EC, G-JD, ME, GF, EJ, MH, RH, EJ, TK, MM-C, PM, ALN, DP, JS-P, AS, ES, SS, HT, PV and BW participated in the study design, piloting and data collection. GT participated in the study design. SH coded some of the data. ME and $\mathrm{SH}$ assisted in validated the coding. ME, MH, RH, TK, ALN, DP and HT worked together to agree the themes. MH had overall responsibility for the study design, recruitment of local leads, analysis of data and interpretation of results. All authors contributed to the writing and review of the manuscript and approved the final version.

Funding This study received no specific grant from any funding agency in the public, commercial or not-for-profit sectors. ALN is supported by the National Institute for Health Research (NIHR) Imperial Patient Safety Translation Research Centre, with infrastructure support provided by the NIHR Imperial Biomedical Research Centre (BRC).

Competing interests None declared.

Patient consent for publication Not required. 
Ethics approval Ethical approval for the study was given by the University of Bath Research Ethics Approval Committee for Health (approval date: 24 November 2014; REACH reference number: EP 14/15 66). Other countries' study leads either achieved local ethical approval or gave statements that formal ethical approval was not needed in their jurisdictions.

Provenance and peer review Not commissioned; externally peer reviewed.

Data availability statement Data are available on reasonable request.

Open access This is an open access article distributed in accordance with the Creative Commons Attribution Non Commercial (CC BY-NC 4.0) license, which permits others to distribute, remix, adapt, build upon this work non-commercially, and license their derivative works on different terms, provided the original work is properly cited, appropriate credit is given, any changes made indicated, and the use is non-commercial. See: http://creativecommons.org/licenses/by-nc/4.0/.

\section{ORCID iDs}

Michael Harris http://orcid.org/0000-0002-7166-2971

Robert Hoffman http://orcid.org/0000-0002-3053-835X

Nicola Buono http://orcid.org/0000-0001-8797-477X

Peter Murchie http://orcid.org/0000-0001-9968-5991

\section{REFERENCES}

1. Møller $\mathrm{H}$, Linklater KM, Robinson D. A visual summary of the EUROCARE-4 results: a UK perspective. Br J Cancer 2009;101 (Suppl 2):S110-S114.

2. EUROCARE. EUROCARE-5. In: Istituto Nazionale Tumori (Milan lalSdSR, Italy). Milan and Rome, 2014.

3. Maringe C, Walters S, Rachet B, et al. Stage at diagnosis and colorectal cancer survival in six high-income countries: a populationbased study of patients diagnosed during 2000-2007. Acta Oncol 2013;52:919-32

4. Walters S, Maringe C, Coleman MP, et al. Lung cancer survival and stage at diagnosis in Australia, Canada, Denmark, Norway, Sweden and the UK: a population-based study, 2004-2007. Thorax 2013;68:551-64.

5. Richards MA. The size of the prize for earlier diagnosis of cancer in England. Br J Cancer 2009;101 (Suppl 2):S125-S129.

6. Woods LM, Coleman MP, Lawrence G, et al. Evidence against the proposition that "UK cancer survival statistics are misleading": simulation study with National Cancer Registry data. BMJ 2011;342. d3399.(doi).

7. Zahl P-H, Jørgensen KJ, Gøtzsche PC. Overestimated lead times in cancer screening has led to substantial underestimation of overdiagnosis. Br J Cancer 2013;109:2014-9. Epub 13 Aug 20

8. Carter JL, Coletti RJ, Harris RP. Quantifying and monitoring overdiagnosis in cancer screening: a systematic review of methods. BMJ 2015;350.

9. Peto J. Cancer epidemiology in the last century and the next decade. Nature 2001:411:390-5.

10. Popic T, Schneider SM. An East-West comparison of healthcare evaluations in Europe: do institutions matter? J Eur Soc Policy 2018;28:517-34.

11. Boscoe FP, Henry KA, Sherman RL, et al. The relationship between cancer incidence, stage and poverty in the United States. Int J Cancer 2016;139:607-12.

12. Schrijvers CT, Mackenbach JP, Lutz JM, et al. Deprivation and survival from breast cancer. Br J Cancer 1995;72:738-43.

13. MacKie RM, Hole DJ. Incidence and thickness of primary tumours and survival of patients with cutaneous malignant melanoma in relation to socioeconomic status. BMJ 1996;312:1125-8.

14. Butler J, Foot C, Bomb M, et al. The International cancer benchmarking partnership: an international collaboration to inform cancer policy in Australia, Canada, Denmark, Norway, Sweden and the United Kingdom. Health Policy 2013;112:148-55.

15. Green T, Atkin K, Macleod U. Cancer detection in primary care: insights from general practitioners. Br J Cancer 2015;112 (Suppl 1:S41-S49.

16. Jacobsen MM, Silverstein SC, Quinn M, et al. Timeliness of access to lung cancer diagnosis and treatment: a scoping literature review. Lung Cancer 2017;112:156-64.

17. McLaughlin JM, Anderson RT, Ferketich AK, et al. Effect on survival of longer intervals between confirmed diagnosis and treatment initiation among low-income women with breast cancer. $\mathrm{J}$ Clin Oncol 2012;30:4493-500.

18. Yun YH, Kim YA, Min YH, et al. The influence of hospital volume and surgical treatment delay on long-term survival after cancer surgery. Ann Oncol 2012;23:2731-7.
19. Gobbi PG, Bergonzi M, Comelli M, et al. The prognostic role of time to diagnosis and presenting symptoms in patients with pancreatic cancer. Cancer Epidemiol 2013;37:186-90.

20. Tørring ML, Frydenberg M, Hansen RP, et al. Evidence of increasing mortality with longer diagnostic intervals for five common cancers: a cohort study in primary care. Eur $J$ Cancer 2013;49:2187-98.

21. Elit LM, O'Leary EM, Pond GR, et al. Impact of wait times on survival for women with uterine cancer. J Clin Oncol 2014;32:27-33.

22. Nanthamongkolkul K, Hanprasertpong J. Longer waiting times for early stage cervical cancer patients undergoing radical hysterectomy are associated with diminished long-term overall survival. J Gynecol Oncol 2015;26:262-9.

23. Neal RD, Tharmanathan P, France B, et al. Is increased time to diagnosis and treatment in symptomatic cancer associated with poorer outcomes? systematic review. Br J Cancer 2015;112 (Suppl 1):S92-S107.

24. Olsson JK, Schultz EM, Gould MK. Timeliness of care in patients with lung cancer: a systematic review. Thorax 2009;64:749-56.

25. Malmström M, Rasmussen BH, Bernhardson B-M, et al. It is important that the process goes quickly, isn't it?" A qualitative multi-country study of colorectal or lung cancer patients' narratives of the timeliness of diagnosis and quality of care. Eur $J$ Oncol Nurs 2018;34:82-8.

26. Foot C, Harrison T. How to improve cancer survival: explaining England's relatively poor rates. London: The King's Fund, 2011.

27. Harris M, Frey P, Esteva M, et al. How health system factors influence referral decisions in patients that may have cancer: European symposium report. J Cancer Res Ther 2016;4:7-10.

28. Johansen M-L, Holtedahl KA, Rudebeck CE. A doctor close at hand: how GPs view their role in cancer care. Scand J Prim Health Care 2010;28:249-55.

29. Adams E, Boulton M, Rose P, et al. Views of cancer care reviews in primary care: a qualitative study. Br J Gen Pract 2011;61:e173-82.

30. Mitchell GK, Burridge LH, Colquist SP, et al. General practitioners' perceptions of their role in cancer care and factors which influence this role. Health Soc Care Community 2012;20:607-16.

31. Perkins N, Coleman A, Wright $M$, et al. The 'added value' GPs bring to commissioning: a qualitative study in primary care. $\mathrm{Br} J$ Gen Pract 2014;64:e728-34.

32. Green T, Atkin K, Macleod U. Gps' perceptions and experiences of public awareness campaigns for cancer: a qualitative enquiry. Health Expect 2016;19:377-87.

33. Harris M, Taylor G. How health system factors affect primary care practitioners' decisions to refer patients for further investigation: protocol for a pan-European ecological study. BMC Health Serv Res 2018;18:338.

34. Center for Aging in Diverse Communities. Guidelines for translating surveys in cross-cultural research. San Francisco: University of California, 2007.

35. Maneesriwongul W, Dixon JK. Instrument translation process: a methods review. J Adv Nurs 2004;48:175-86.

36. Harris M, Vedsted P, Esteva M, et al. Identifying important health system factors that influence primary care practitioners' referrals for cancer suspicion: a European cross-sectional survey. BMJ Open 2018;8:e022904.

37. Patton M. Qualitative Research \& Evaluation Methods. 177. Beverly Hills: Sage, 1990.

38. Braun V, Clarke V. Using thematic analysis in psychology. Qual Res Psychol 2006;3:77-101.

39. Daly $\mathrm{H}$, Collins $\mathrm{C}$. Barriers to early diagnosis of cancer in primary care: a needs assessment of GPs. Ir Med J 2007;100:624-6.

40. Car LT, Papachristou N, Urch C, et al. Preventing delayed diagnosis of cancer: clinicians' views on main problems and solutions. J Glob Health 2016;6:020901.

41. Ersin F, Bahar Z. Barriers and facilitating factors perceived in Turkish women's behaviors towards early cervical cancer detection: a qualitative approach. Asian Pac J Cancer Prev 2013;14:4977-82.

42. Cassim S, Chepulis L, Keenan R, et al. Patient and carer perceived barriers to early presentation and diagnosis of lung cancer: a systematic review. BMC Cancer 2019;19:25.

43. Kislov R, Walshe K, Harvey G. Managing boundaries in primary care service improvement: a developmental approach to communities of practice. Implement Sci 2012;7.

44. Cook N, Hart A, Nuttall K, et al. A telephone survey of cancer awareness among frontline staff: informing training needs. $\mathrm{Br} \mathrm{J}$ Cancer 2011;105:340-5.

45. Cook N, Thomson G, Dey P. Managing risk in cancer presentation, detection and referral: a qualitative study of primary care staff views. BMJ Open 2014;4:e004820. 
46. Rubin G, Vedsted P, Emery J. Improving cancer outcomes: better access to diagnostics in primary care could be critical. $\mathrm{Br} J$ Gen Pract 2011;61:317-8.

47. Rubin GP, Saunders CL, Abel GA, et al. Impact of investigations in general practice on timeliness of referral for patients subsequently diagnosed with cancer: analysis of national primary care audit data. Br J Cancer 2015;112:676-87.

48. Jiwa M, Meng X, O'Shea C, et al. How do general practitioners manage patients with cancer symptoms? A video-vignette study. BMJ Open 2015;5:e008525. 